\title{
A generalization of Aztec diamond theorem, part I
}

\author{
Tri Lai \\ Department of Mathematics \\ Indiana University \\ Bloomington, IN 47405, U.S.A. \\ tmlai@indiana.edu
}

Submitted: Sep 10, 2013; Accepted: Feb 26, 2014; Published: Mar 10, 2014

Mathematics Subject Classifications: 05A15, 05E99

\begin{abstract}
We consider a new family of 4-vertex regions with zigzag boundary on the square lattice with diagonals drawn in. By proving that the number of tilings of the new regions is given by a power 2, we generalize both Aztec diamond theorem and Douglas' theorem. The proof extends an idea of Eu and Fu for Aztec diamonds, by using a bijection between domino tilings and non-intersecting Schröder paths, then applying Lindström-Gessel-Viennot methodology.
\end{abstract}

Keywords: Aztec diamonds, dominos, tilings, perfect matchings, Schröder paths

\section{Introduction}

Given a lattice in the plane, a (lattice) region is a finite connected union of fundamental regions of that lattice. A tile is the union of two fundamental regions sharing an edge. A tiling of the region $R$ is a covering of $R$ by tiles so that there are no gaps or overlaps.

A perfect matching of a graph $G$ is a collection of edges such that each vertex of $G$ is adjacent to precisely one edge in the collection. Denote by $\mathrm{M}(G)$ the number of perfect matchings of graph $G$. The tilings of a region $R$ can be naturally identified with the perfect matchings of its dual graph (i.e., the graph whose vertices are the fundamental regions of $R$, and whose edges connect two fundamental regions precisely when they share an edge). In the view of this, we denote by $\mathrm{M}(R)$ the number of tilings of $R$.

The Aztec diamond region of order $n$ is defined to be the union of all the unit squares with integral corners $(x, y)$ satisfying $|x|+|y| \leqslant n+1$ in the Cartesian coordinate system (see Figure 1 for an example of Aztec diamond region of order 4). The number of tilings of an Aztec diamond region is given by the following theorem that was first proved by Elkies, Kuperberg, Larsen and Propp [5]. 
Theorem 1 (Aztec diamond theorem [5]). The number of (domino) tilings of the Aztec diamond of order $n$ is $2^{n(n+1) / 2}$.

Further proofs of the Aztec diamond theorem were given by several authors (see e.g., [2], [3], [6], [9], [10], [12]).
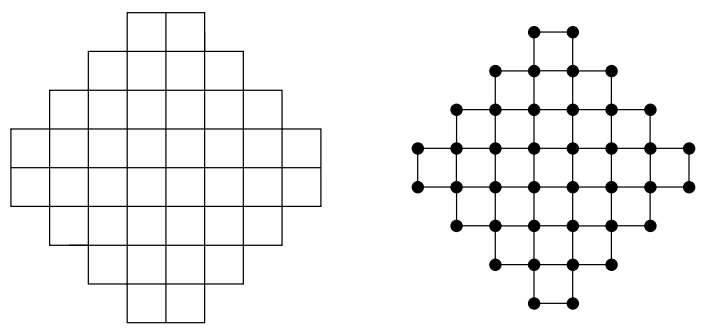

Figure 1: The Aztec diamond region of order 4 (left) and its dual graph, the Aztec diamond graph of order 4 (right).

Douglas [4] considered a certain family of regions in the square lattice with every second southwest-to-northeast diagonal drawn in (examples are shown in Figure 2). Precisely, the region of order $n$, denoted by $D(n)$, has four vertices that are the vertices of a diamond of side-length $2 n \sqrt{2}$.

Theorem 2 (Douglas [4]).

$$
\mathrm{M}(D(n))=2^{2 n(n+1)} .
$$
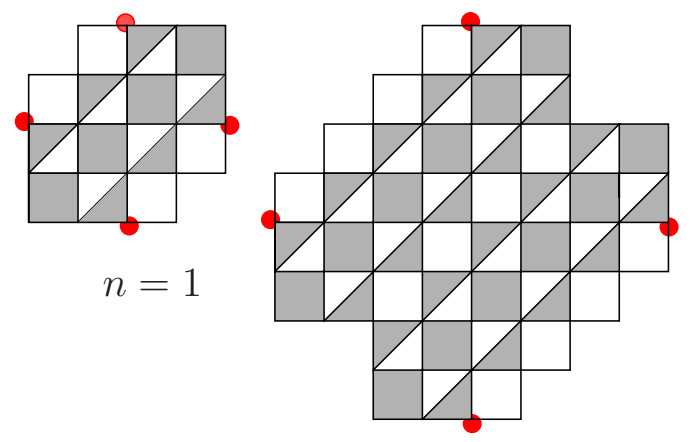

$n=2$

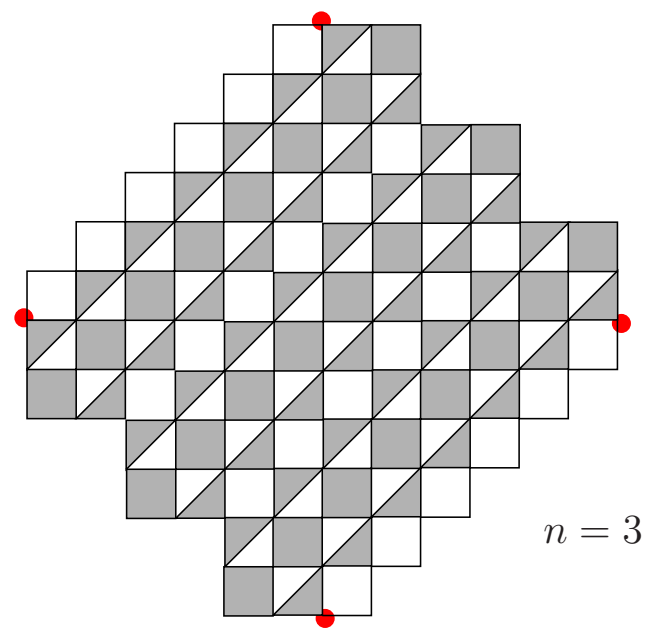

Figure 2: The Douglas' regions of order $n=1, n=2$ and $n=3$.

The regions in the Douglas' theorem have the distances ${ }^{1}$ between any two successive southwest-to-northeast diagonals drawn in are 2. Next, we consider general situation when the distances between two successive drawn-in diagonals ${ }^{2}$ are arbitrary.

\footnotetext{
${ }^{1}$ The unit here is the distance between two consecutive lattice diagonals of the square lattice, i.e. $\sqrt{2} / 2$.

${ }^{2}$ From now on, "diagonal(s)" refers to "southwest-to-northeast diagonal(s)"
} 
Consider the setup of drawn-in diagonals in the square lattice as follows. Let $\ell$ and $\ell^{\prime}$ be two fixed lattice diagonals ( $\ell$ and $\ell^{\prime}$ are not drawn-in diagonals), and assume that $k-1$ diagonals have been drawn in between $\ell$ and $\ell^{\prime}$, with the distances between successive ones, starting from top, being $d_{2}, \ldots, d_{k-2}$. The distance between $\ell$ and the top drawn-in diagonal is $d_{1}$, and the distance between the bottom drawn-in diagonal and $\ell^{\prime}$ is $d_{k}$.

Given a positive integer $a$, we define the region $D_{a}\left(d_{1}, \ldots, d_{k}\right)$ as follows (see Figure 3 for an example). Its southwestern and northeastern boundaries are defined in the next two paragraphs.

Color the resulting dissection of the square lattice black and white so that any two fundamental regions that share an edge have opposite colors, and assume that the fundamental regions passed through by $\ell$ are white (by definition $\ell$ and $\ell^{\prime}$ pass through unit squares). Let $A$ be a lattice point on $\ell$. Start from $A$ and take unit steps south or east so that for each step the color of the fundamental region on the left is black. We arrive $\ell^{\prime}$ at a lattice point $B$. The described path from $A$ to $B$ is the northeastern boundary of our region.

Let $D$ be the lattice point on $\ell$ that is a unit square diagonals to the southwest of $A$ (i.e. $|A D|=a \sqrt{2}$ ). The southwestern boundary is obtained from the northeastern boundary by reflecting it about the perpendicular bisector of segment $A D$, and reversing the directions of its unit steps (from south to north, and from east to west). Let $C$ be the reflection point of $B$ about the perpendicular bisector above, so $C$ is also on $\ell^{\prime}$.

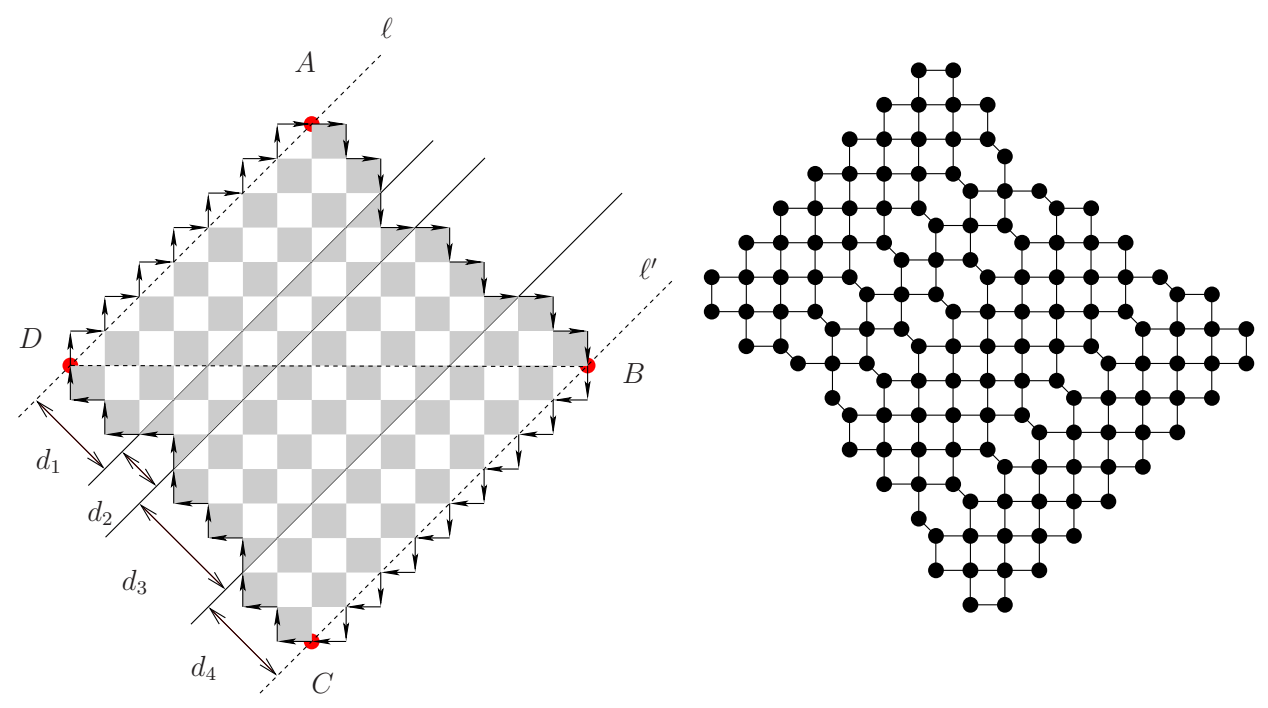

Figure 3: The region $D_{7}(4,2,5,4)$ (left) and its dual graph (right).

Connect $D$ and $A$ by a zigzag lattice path consisting of alternatively east and north steps, so that the unit squares passed through by $\ell$ are on the right of the zigzag path. Similarly, we connect $B$ and $C$ by a zigzag lattice path, so that the square cells passed through by $\ell^{\prime}$ are on the right. These two zigzag lattice paths are northwestern and southeastern boundaries, and they complete the boundary of the region $D_{a}\left(d_{1}, \ldots, d_{k}\right)$. We call the resulting region a generalized Douglas region. 
Remark 3. (1) If the line $\ell^{\prime}$ passes through black unit squares, then the region does not have a tiling (since we can not cover the black squares by disjoint tiles). Hereafter, we assume that $\ell^{\prime}$ passes through white unit square.

(2) Since we only consider connected region, we also assume from now on that the southwestern and northeastern boundaries do not intersect each other.

We call the fundamental regions in a generalized Douglas region cells. Note that there are two kinds of cells, square and triangular. The latter in turn come in two orientations: they may point towards $\ell^{\prime}$ or away from $\ell^{\prime}$. We call them down-pointing triangles or up-pointing triangles, respectively. A cell is said to be regular if it is a black square or a black up-pointing triangle.

A (southwest-to-northeast) line of cells consists of all the triangular cells of a given color with bases resting on a fixed lattice diagonal, or consists of all the square cells (of a given color) passed through by a fixed lattice diagonal. Define the width of our region to be the number of white squares in the bottom line of cells. One readily sees that the width of the region is exactly $|B C| / \sqrt{2}$, where $|B C|$ is the Euclidian distance between $B$ and $C$. The number of tilings of a generalized Douglas region is obtained by the theorem stated below.

Theorem 4. Assume that $a, d_{1}, \ldots, d_{k}$ are positive integers, so that for which the generalized Douglas region $D_{a}\left(d_{1}, \ldots, d_{k}\right)$ has the width $w$, and has its western and eastern vertices (i.e. the vertices $B$ and $D$ ) on the same horizontal line. Then

$$
\mathrm{M}\left(D_{a}\left(d_{1}, \ldots, d_{k}\right)\right)=2^{\mathcal{C}-w(w+1) / 2}
$$

where $\mathcal{C}$ is the number of regular cells in the region.

Let $k=1$ (i.e. there are no dawn-in diagonals between $\ell$ and $\ell^{\prime}$ ) and $a=d_{1}=n$, our generalized Douglas region, $D_{a}\left(d_{1}, \ldots, d_{k}\right)$, is exactly the Aztec diamond region of order $n$. One readily sees that the region has the width $w=n$ and the number of regular cells $\mathcal{C}=n(n+1)$. This means that we can imply Aztec diamond theorem 1 from Theorem 4 .

Moreover, one can get Douglas' theorem 2 from the Theorem 4 by setting $k=2 n \geqslant 2$, $d_{1}=d_{k}=1, a=k$, and $d_{2}=d_{3}=\ldots=d_{k-1}=2$. Therefore, Theorem 4 can be view as a common multi-parameter generalization of Aztec diamond theorem and Douglas' theorem.

For the sake of simplicity, hereafter, "square(s)" refers to "square cell(s)", and "triangle(s)" refers to "triangular cells".

The goal of this paper is to prove Theorem 4. Our proof is inspired by the idea of $\mathrm{Eu}$ and $\mathrm{Fu}$ in [6]. In particular, the proof uses some one-to-one correspondence between domino tilings and barred non-intersecting Schröder paths, then applies Lindström-GesselViennot lemma (see Lemma 5).

\section{Structure of generalized Douglas regions}

Our goal of this section is to investigate further the structure of generalized Douglas regions. 
Consider a generalized Douglas region $D_{a}\left(d_{1}, \ldots, d_{k}\right)$. Denote by $p$ the number of (southwest-to-northeast) lines of black square cells, denote by $q$ the number of lines of black up-pointing triangular cells, and denote by $l$ the number of lines of black downpointing triangular cells in the region.

The region $D_{a}\left(d_{1}, \ldots, d_{k}\right)$ can be partitioned into $a$ horizontal strips of cells above $B D$ and $w$ horizontal strips of cells below $B D$ (see Figure 4 for an example with $a=7, k=4$, $\left.d_{1}=4, d_{2}=2, d_{3}=5, d_{4}=4\right)$. Consider the horizontal strips above segment $B D$. Each of them starts by a white square in the top line of cells, and ends by a black square or a black down pointing-triangle along the northeastern boundary of the region. Compare the number of starting cells and the number of ending cells in those strips, we get

$$
a=p+l \text {. }
$$

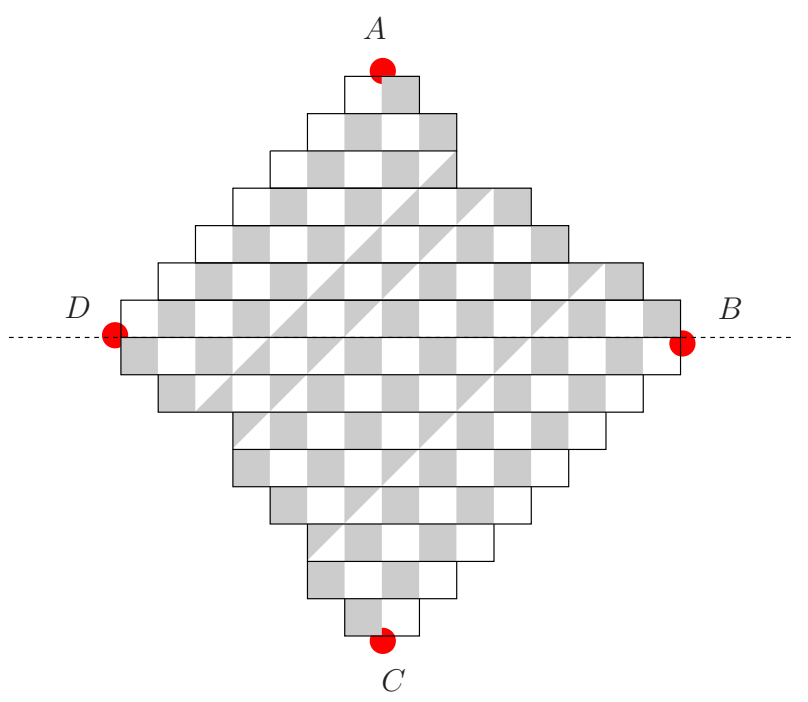

Figure 4: Partitioning a generalized Douglas region into horizontal strips of cells.

We consider now the horizontal strips below the segment $B D$. Each of them starts by a black square or a black up-pointing triangle along the southwestern boundary, and ends by a white square in the bottom lines of cells. Again, we compare of the number of starting cells and the number of ending cells in those strips, and obtain

$$
w=p+q
$$

From (3) and (4), we get

$$
\begin{gathered}
a+q-l=p+q=w, \\
a+w=2 p+q+l .
\end{gathered}
$$

Consider the number of unit steps on the southwestern boundary of the region. Each line of black squares contributes 2 steps, and each line of black triangles contributes 1 step to the latter number of steps. Thus, the number of steps here is exactly the expression on 
the right hand side of $(5 \mathrm{~b})$. On the other hand, one readily see that the number of steps on the southwestern boundary is equal to the sum of all distances $d_{i}$ 's. Therefore,

$$
a+w=2 p+q+l=\sum_{i=1}^{k} d_{i} .
$$

For each of $k-1$ drawn-in diagonals of $D_{a}\left(d_{1}, \ldots, d_{k}\right)$, there is exactly one line of black up-pointing triangles or one line of black down-pointing triangles with bases resting on it. This implies that the number of lines of black triangles is equal to $k-1$, i.e.

$$
q+l=k-1 .
$$

The $k-1$ drawn-in diagonals divide the region into $k$ parts called layers. The first layer is the part above the top drawn-in diagonal, the last layer is the part below the bottom drawn-in diagonal, and the $i$-th layer (for $1<i<k$ ) is the part between the $(i-1)$-th and the $i$-th drawn-in diagonals.

\section{$3 \quad$ Schröder paths with barriers}

A Schröder path is a path in the plane, starting and ending on the $x$-axis, never going below the $x$-axis, using $(1,1),(1,-1)$ and $(2,0)$ steps (i.e. up, down and level steps, respectively). Denote by $\mathrm{U}, \mathrm{D}$, and $\mathrm{L}$ the up, down and level steps, respectively.

A barrier is a length-1 horizontal segment in the plane. A Schröder path is said to be compatible with a setup of barriers if it does not cross any barriers of the setup.

Let $a_{1}, \ldots, a_{m}$ be nonnegative integers so that $a_{1}<a_{2}<\ldots<a_{m}$. We consider a setup of barriers as follows. For any $k \in \mathbb{Z}$ and $1 \leqslant i \leqslant m$, we draw a barrier connecting two points $\left(-a_{i}+k, k+\frac{1}{2}\right)$ and $\left(-a_{i}+k+1, k+\frac{1}{2}\right)$ (i.e. all barriers appear along the lines $y=x+a_{i}$, for $\left.i=1,2, \ldots, m\right)$. Denote by $\operatorname{Bar}\left(a_{1}, a_{2}, \ldots, a_{m}\right)$ the resulting setup of barriers. A bad level step (with respect to the setup $\operatorname{Bar}\left(a_{1}, a_{2}, \ldots, a_{m}\right)$ ) of a Schröder path is a level step from $(x, 0)$ to $(x+2,0)$, where $x \notin\left\{-a_{i}-1: 1 \leqslant i \leqslant m\right\}$. Figure 5 illustrates an example of a Schröder path compatible with the set of barriers $\operatorname{Bar}(2,5,8)$, and the path has a bad level step from $(1,0)$ to $(3,0)$.

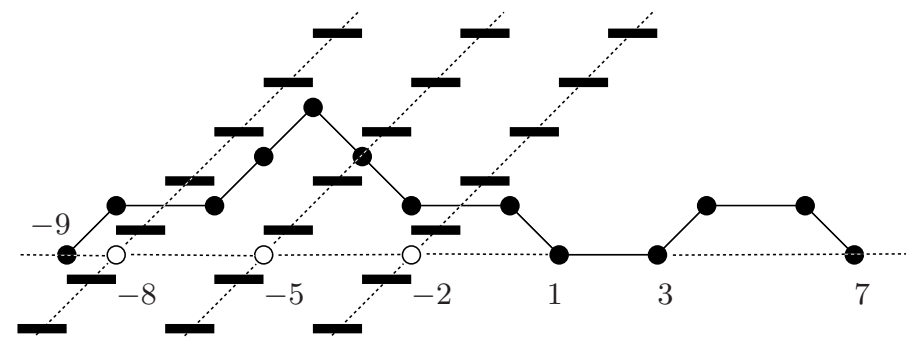

Figure 5: A Schröder path compatible with barrier set $\operatorname{Bar}(2,5,8)$.

Let $x_{i}$ be the $i$-th largest negative odd number in $\mathbb{Z} \backslash\left\{-a_{1}, \ldots,-a_{m}\right\}$, let $A_{i}$ be the point $\left(x_{i}, 0\right)$, and let $B_{i}$ be the point $(2 i-1,0)$, for $i=1,2, \ldots, n$. We consider two 
sets of $n$-tuples of non-intersecting Schröder paths compatible with $\operatorname{Bar}\left(a_{1}, a_{2}, \ldots, a_{m}\right)$ as follows.

The set $\Pi_{n}\left(a_{1}, \ldots, a_{m}\right)$ consists of $n$-tuples of non-intersecting Schröder paths $\left(\pi_{1}, \pi_{2}\right.$, $\left.\ldots, \pi_{n}\right)$ (compatible with $\operatorname{Bar}\left(a_{1}, a_{2}, \ldots, a_{m}\right)$ ), where $\pi_{i}$ connects two points $A_{i}$ and $B_{i}$. The set $\Lambda_{n}\left(a_{1}, \ldots, a_{m}\right)$ consists of $n$-tuples of non-intersecting Schröder paths $\left(\lambda_{1}, \lambda_{2}, \ldots\right.$, $\lambda_{n}$ ) (compatible with $\operatorname{Bar}\left(a_{1}, a_{2}, \ldots, a_{m}\right)$ ), where $\lambda_{i}$ connects $A_{i}$ and $B_{i}$, and has no bad level steps.

The following well-known result is due to Lindström-Gessel-Viennot (see [7]; [11], Lemma 1; [13] Theorem 1.2).

Lemma 5. Let $U=\left(u_{1}, u_{2}, \ldots, u_{n}\right)$ and $V=\left(v_{1}, v_{2}, \ldots, v_{n}\right)$ be two $n$-tuples of vertices in an acyclic digraph $G$. Assume that for any $1 \leqslant i<i^{\prime} \leqslant n$ and $\left.n \geqslant j\right\rangle j^{\prime} \geqslant 1$, every path $P \in \mathcal{P}\left(u_{i}, v_{j}\right)$ intersects every path $Q \in \mathcal{P}\left(u_{i^{\prime}}, v_{j^{\prime}}\right)$, where $\mathcal{P}\left(u_{i}, v_{j}\right)$ (resp., $\mathcal{P}\left(u_{i^{\prime}}, v_{j^{\prime}}\right)$ ) is the set of paths in $G$ from $u_{i}$ to $v_{j}$ (resp., from $u_{i^{\prime}}$ to $v_{j^{\prime}}$ ). Then the number of $n$-tuples of non-intersecting paths connecting vertices in $U$ to vertices in $V$ is equal to $\operatorname{det}\left(\left(a_{i, j}\right)_{1 \leqslant i, j \leqslant n}\right)$, where $a_{i, j}$ is the number of paths in $G$ from $u_{i}$ to $v_{j}$.

Given a setup of barriers $\operatorname{Bar}\left(a_{1}, \ldots, a_{m}\right)$, where $a_{1}<a_{2}<\ldots<a_{m}$. We define

$$
H_{n}:=H_{n}\left(a_{1}, \ldots, a_{m}\right):=\left[\begin{array}{cccc}
r_{1,1} & r_{1,2} & \ldots & r_{1, n} \\
r_{2,1} & r_{2,2} & \ldots & r_{2, n} \\
\vdots & \vdots & & \vdots \\
r_{n, 1} & r_{n, 2} & \ldots & r_{n, n}
\end{array}\right] \text {, }
$$

and

$$
G_{n}:=G_{n}\left(a_{1}, \ldots, a_{m}\right):=\left[\begin{array}{cccc}
s_{1,1} & s_{1,2} & \ldots & s_{1, n} \\
s_{2,1} & s_{2,2} & \ldots & s_{2, n} \\
\vdots & \vdots & & \vdots \\
s_{n, 1} & s_{n, 2} & \ldots & s_{n, n}
\end{array}\right],
$$

where $r_{i, j}$ (resp., $s_{i, j}$ ) is the number of Schröder paths (resp., Schröder paths without bad level steps) from $A_{i}$ to $B_{j}$, where $A_{i}=\left(x_{i}, 0\right)$ with $x_{i}$ is the $i$ th largest negative odd integer in $\mathbb{Z} \backslash\left\{-a_{1}, \ldots,-a_{m}\right\}$, and where $B_{j}=(2 j-1,0)$, for $1 \leqslant i, j \leqslant n$.

Proposition 6. For any positive integers $n$ and $m$, we have

$$
\begin{aligned}
\left|\Pi_{n}\left(a_{1}, \ldots, a_{m}\right)\right| & =\operatorname{det}\left(H_{n}\left(a_{1}, \ldots, a_{m}\right)\right), \\
\left|\Lambda_{n}\left(a_{1}, \ldots, a_{m}\right)\right| & =\operatorname{det}\left(G_{n}\left(a_{1}, \ldots, a_{m}\right)\right) .
\end{aligned}
$$

Proof. Consider two sets of points: $\mathcal{A}=\left\{A_{1}, \ldots, A_{n}\right\}$ and $\mathcal{B}=\left\{B_{1}, \ldots, B_{n}\right\}$, where $A_{i}$ 's and $B_{j}$ 's are defined as in the paragraph before the statement of the theorem.

Let $G$ be the digraph defined as follows. The vertex set of $G$ consists of all lattice points of the square lattice that are inside or on the edges of the up-pointing isosceles right triangle whose hypothenuse is segment $A_{n} B_{n}$, and that can be reached from $A_{n}$ by 
$(1,1),(1,-1)$ and $(2,0)$ steps. An edge of $G$ connects from $(x, y)$ to $\left(x^{\prime}, y^{\prime}\right)$ if we can go from the former vertex to the latter vertex by one of the above steps. Next, we remove all edges which cross some barriers of $\operatorname{Bar}\left(a_{1}, \ldots, a_{m}\right)$ (see the illustrative picture in Figure $6(\mathrm{a})$, for $m=3, n=4, a_{1}=2, a_{2}=5$, and $\left.a_{3}=8\right)$.

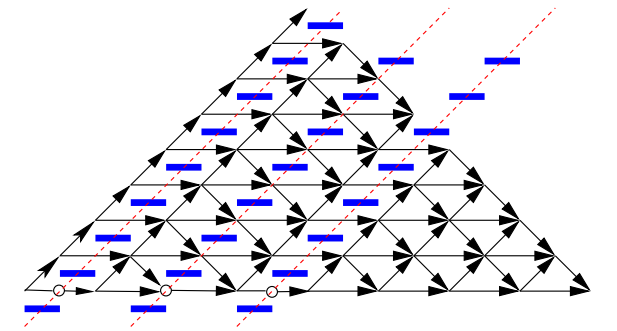

(a)

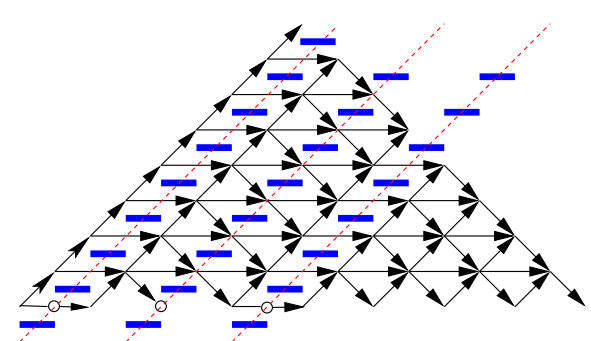

(b)

Figure 6: Illustrating the proof of Proposition 6.

Apply the Lemma 5 to the digraph $G$ with two compatible sets of vertices $\mathcal{A}$ and $\mathcal{B}$, we get

$$
\operatorname{det}\left(H_{n}\right)=\left|\Pi_{n}\left(a_{1}, \ldots, a_{m}\right)\right|
$$

which proves (10).

The equality (11) can be proved similarly. We apply the same procedure as in the proof of (10) to the digraph $G^{\prime}$ that is obtained from the graph $G$ by removing all horizontal edges on $x$-axis containing no points $\left(-a_{i}, 0\right)$, for $1 \leqslant i \leqslant m$ (see Figure $6(\mathrm{~b})$ ).

Similar to the relationship between large and small Schröder numbers (see [6] and [8]), we have the following fact about $r_{i, j}$ and $s_{i, j}$.

Proposition 7. Given a setup of barriers $\operatorname{Bar}\left(a_{1}, \ldots, a_{m}\right)$. If $a_{1}>1$, then $r_{i, j}=2 s_{i, j}$, for any $1 \leqslant i, j \leqslant n$.

Proof. It is easy to see $r_{1,1}=2=2 s_{1,1}$. Thus, we assume in the rest of the proof that $i+j \geqslant 3$.

Fix two indices $i$ and $j$, so that $i+j \geqslant 3$. We consider the following two subsets of the set of all Schröder paths from $A_{i}$ to $B_{j}$, which are compatible with $\operatorname{Bar}\left(a_{1}, \ldots, a_{m}\right)$ :

(i) The set $S$ of the paths having at least one bad level step;

(ii) The set $S^{\prime}$ of the paths having no bad level steps.

We have a bijection between $S$ and $S^{\prime}$ working as follows.

Let $\tau$ be a Schröder path in $S$. We can factor $\tau=P \mathrm{~L} Q$, where $\mathrm{L}$ is the last bad level step in $\tau$, so $Q$ has no bad level steps (see the upper picture in Figure 7 ). We define a Schröder path $\lambda=\mathrm{UPDQ}$ (see the lower picture in Figure 7 ). One readily sees that $\lambda$ is compatible with the setup of barriers $\operatorname{Bar}\left(a_{1}, \ldots, a_{m}\right)$, and has no bad level steps. It means $\lambda \in S^{\prime}$. Since $\lambda$ is determined uniquely by $\tau$, this gives an injection from $S$ to $S^{\prime}$. 

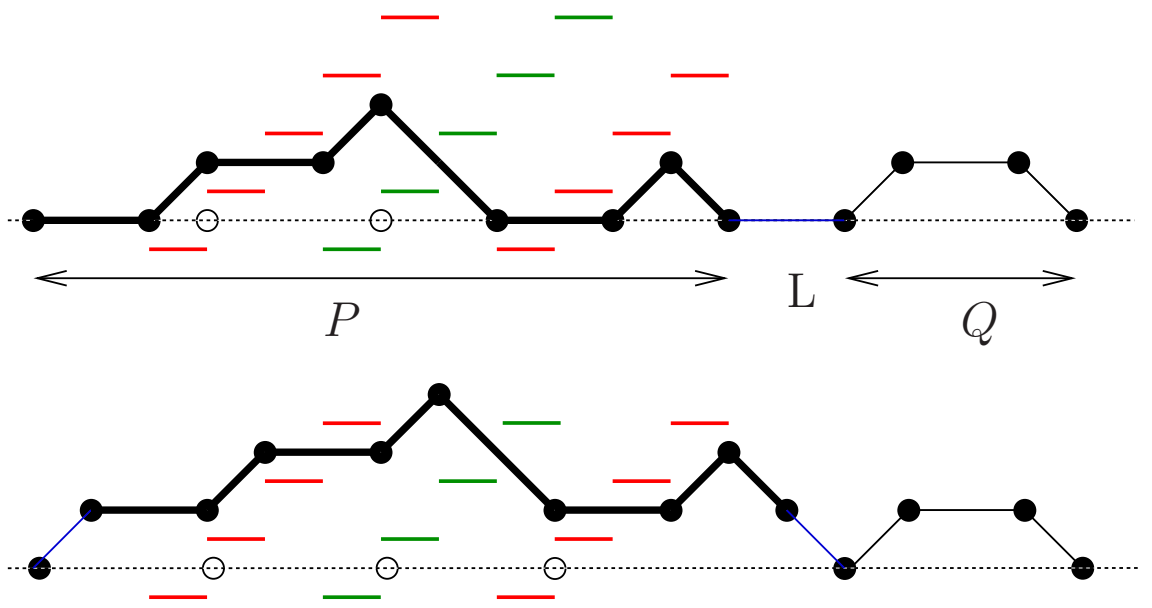

Figure 7: A bijection between two types of Schröder paths with barriers.

On the other hand, let $\lambda$ be a Schröder path in $S^{\prime}$, and let $A^{*}=(c, 0)$ the first returning point of $\lambda$ to $x$-axis. We factor $\lambda=\bar{\lambda} Q$, where $\bar{\lambda}$ is the portion of $\lambda$ connecting $A_{i}$ and $A^{*}$. We can factor further $\bar{\lambda}=\mathrm{U} P \mathrm{D}$ by the choice of $A^{*}$. Next, we define a Schröder path $\tau=P L Q$. We have the number $c-2$ is not in the set $\left\{-a_{i}-1: 1 \leqslant i \leqslant m\right\}$ (otherwise the last step of $\bar{\lambda}$, which is a down step, crosses a barrier, a contradiction). Thus, by definition, the level step $\mathrm{L}$, from $(c-2,0)$ to $(c, 0)$, in the factorization of $\tau$ is a bad level step. Moreover, $\tau$ is compatible with $\operatorname{Bar}\left(a_{1}, \ldots, a_{m}\right)$, so $\tau \in S$. Since $\tau$ is determined uniquely by $\lambda$, this yields an injection from $S^{\prime}$ to $S$.

Therefore, we have a bijection between $S$ and $S^{\prime}$, which completes the proof of the lemma.

Proposition 8. For any positive integers $n, m$, and for any nonnegative integers $a_{1}, a_{2}$, $\ldots, a_{m}$ so that $a_{1}<a_{2}-1<\ldots<a_{m}-1$

(a) $\left|\Pi_{n}\left(a_{1}, \ldots, a_{m}\right)\right|=\left|\Lambda_{n+1}\left(a_{1}+2, \ldots, a_{m}+2\right)\right|$.

(b) $\left|\Lambda_{n}\left(1, a_{2} \ldots, a_{m}\right)\right|=\left|\Pi_{n}\left(a_{2}-2, \ldots, a_{m}-2\right)\right|$ if $a_{1}=1$.

(c) $\left|\Pi_{n}\left(0, a_{2} \ldots, a_{m}\right)\right|=\left|\Pi_{n-1}\left(a_{2}-2 \ldots, a_{m}-2\right)\right|$ if $a_{1}=0$.

Proof. (a) We have a bijection $\varphi$ between two sets $\Pi_{n}\left(a_{1}, \ldots, a_{m}\right)$ and $\Lambda_{n+1}\left(a_{1}+2, \ldots, a_{m}+\right.$ $2)$ defined as follows. $\varphi$ carries $\left(\pi_{1}, \ldots, \pi_{n}\right) \in \Pi_{n}\left(a_{1}, \ldots, a_{m}\right)$ into $\left(\lambda_{1}, \ldots, \lambda_{n+1}\right) \in$ $\Lambda_{n+1}\left(a_{1}+2, \ldots, a_{m}+2\right)$, where $\lambda_{1}=\mathrm{UD}$ and $\lambda_{i+1}=\mathrm{U}^{(i-1)} \pi_{i} \mathrm{D}^{(i-1)}$, for $1 \leqslant i \leqslant n$. This bijection is illustrated in Figure 8.

(b) There is also a bijection $\psi$ between $\Pi_{n}\left(a_{2}-2 \ldots, a_{m}-2\right)$ and $\Lambda_{n}\left(1, a_{2} \ldots, a_{m}\right)$ by setting

$$
\psi\left(\left(\pi_{1}, \ldots, \pi_{n}\right)\right)=\left(\lambda_{1}, \ldots, \lambda_{n}\right) \in \Lambda_{n}\left(1, a_{1}, \ldots, a_{m}\right),
$$

where $\lambda_{i}:=\mathrm{U} \pi_{i} \mathrm{D}$, for $1 \leqslant i \leqslant n$. This bijection is illustrated in Figure 9.

(c) We construct a bijection $\phi$ between two sets $\Pi_{n}\left(0, a_{2} \ldots, a_{m}\right)$ and $\Pi_{n-1}\left(a_{2}-\right.$ $\left.2 \ldots, a_{m}-2\right)$, for $n \geqslant 2$, as follows. Let $\left(\pi_{1}, \ldots, \pi_{n-1}\right)$ be an element of $\Pi_{n-1}\left(a_{2}-\right.$ $\left.2 \ldots, a_{m}-2\right)$. 


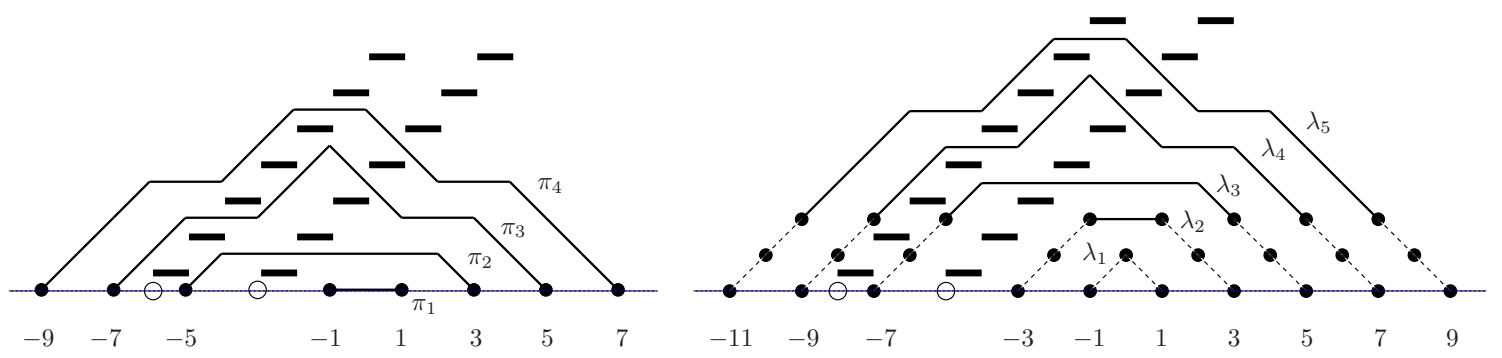

Figure 8: Illustrating the proof of Lemma $8(\mathrm{a})$, for $m=2, n=4, a_{1}=3$, and $a_{2}=6$.

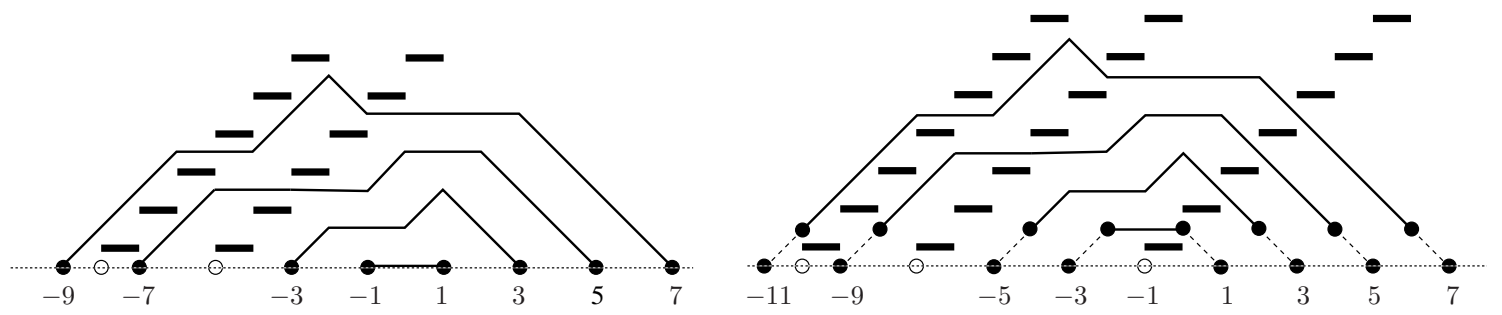

Figure 9: Illustrating the proof of Lemma $8(\mathrm{~b})$, for $m=3, n=4, a_{1}=1, a_{2}=7$ and $a_{3}=10$.

It is easy to see that the last $i-1$ steps of $\pi_{i}$ are down steps, for $2 \leqslant i \leqslant n-1$. Thus, we can factor $\pi_{i}:=\widetilde{\pi}_{i} \mathrm{D}^{(i-1)}$, for $2 \leqslant i \leqslant n-1$. Let $\pi_{1}^{\prime}:=\mathrm{L}, \pi_{2}^{\prime}:=\mathrm{U} \pi_{1} \mathrm{~L} \mathrm{D}$ and $\pi_{i}^{\prime}:=\mathrm{U} \tilde{\pi}_{i-1} \mathrm{LD} \mathrm{D}^{(i-1)}$, for $2 \leqslant i \leqslant n$ (see Figure 10 ). Define $\phi$ by setting

$$
\phi\left(\left(\pi_{1}, \ldots, \pi_{n-1}\right)\right):=\left(\pi_{1}^{\prime}, \ldots, \pi_{n}^{\prime}\right) .
$$

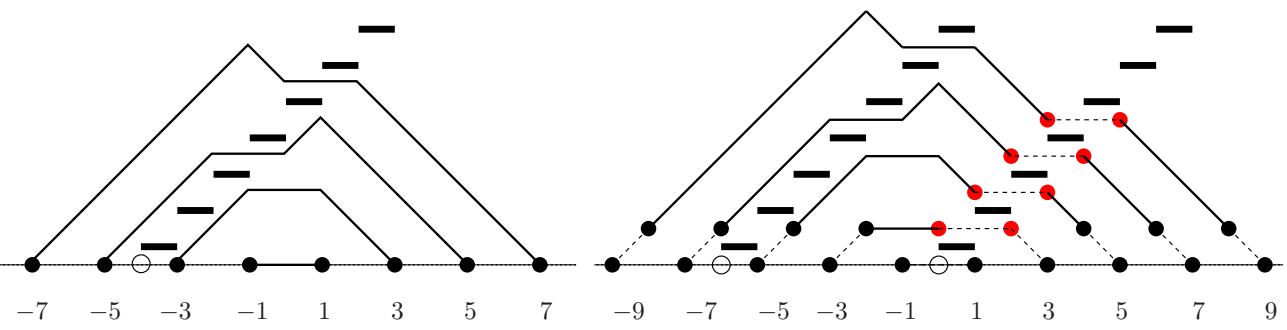

Figure 10: Illustrating the proof of Lemma $8(\mathrm{c})$, for $m=2, n=5, a_{1}=0$, and $a_{2}=6$.

\section{Proof of Theorem 4}

Before presenting the proof of Theorem 4, we prove an important fact stated in the next proposition. 
Proposition 9. Assume that $a, d_{1}, \ldots, d_{k}$ are positive integers so that the generalized Douglas region $D_{a}\left(d_{1}, d_{2}, \ldots, d_{k}\right)$ has the width $w$, and has its western and eastern vertices on the same horizontal line. Let $a_{i}:=d_{k}+\ldots+d_{k-i+1}+i-1$, for $i=1,2, \ldots, k-1$. Then

$$
\mathrm{M}\left(D_{a}\left(d_{1}, \ldots, d_{k}\right)\right)=\left|\Pi_{w}\left(a_{1}, \ldots, a_{k-1}\right)\right| .
$$

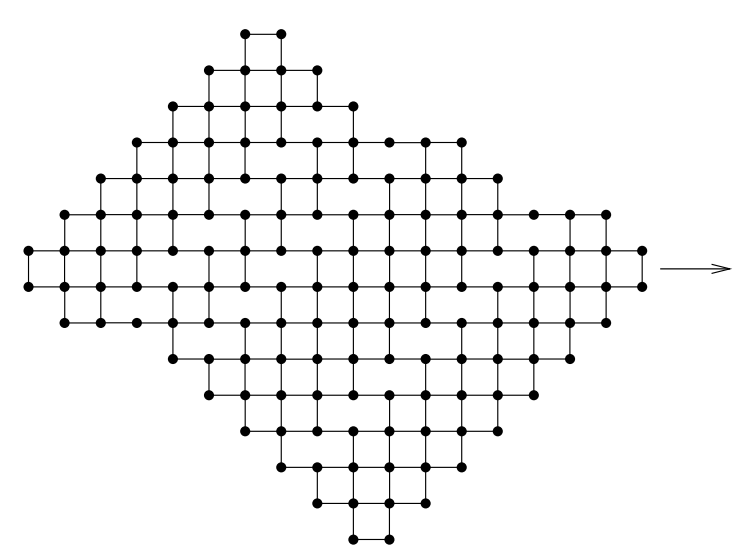

(a)

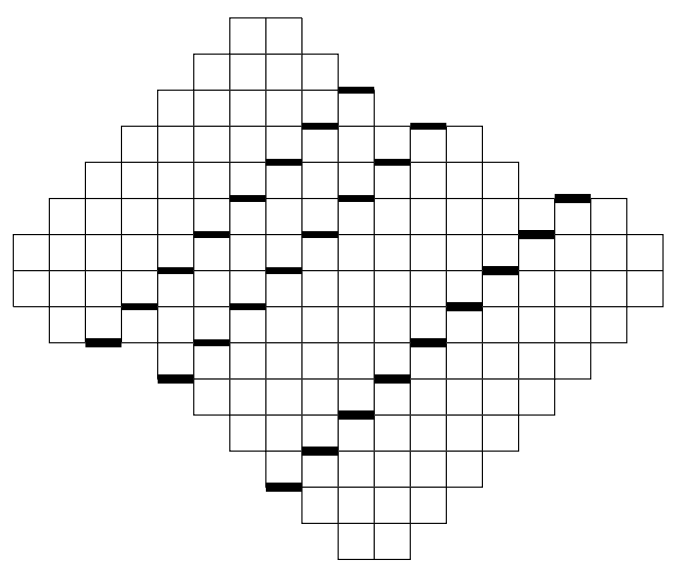

(b)

Figure 11: Transforming the dual graph of $D_{7}(4,2,5,4)$ (after deformed into a subgraph of the square grid) into the region $\bar{D}_{7}(4,2,5,4)$ associating with some bold barriers.

Proof. We first deform the dual graph $G$ of $\mathcal{D}:=D_{a}\left(d_{1}, \ldots, d_{k}\right)$ into a subgraph $G^{\prime}$ of the infinite square grid $\mathbb{Z}^{2}$ (see Figure 11(a) for an example). We notice that $G^{\prime}$ is a planar graph. We apply the local transformation to all square and hexagonal faces as of $G^{\prime}$ in Figure 12. In particular, a square face is replaced by a $2 \times 2$ block, and a hexagonal faces is replaced by a $2 \times 3$ block with 3 barriers. We obtain a new region associating with a sets barriers, denoted by $\overline{\mathcal{D}}:=\bar{D}_{a}\left(d_{1}, \ldots, d_{k}\right)$ (see Figure 11(b)). We notice that if two faces in $G^{\prime}$ have some vertices in common, then their blocks in $\overline{\mathcal{D}}$ share the unit squares (and the barriers) corresponding to the common vertices.
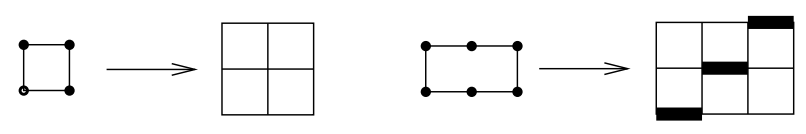

Figure 12: The local replacements in creating $\overline{\mathcal{D}}$ from $G^{\prime}$.

A bad domino is a vertical domino having a middle barrier (see the middle vertical domino of the $2 \times 3$ block in Figure 12), and a tiling of $\overline{\mathcal{D}}$ is good if it contains no bad dominoes. We have

$$
\mathrm{M}\left(G^{\prime}\right)=\mathrm{M}^{*}(\overline{\mathcal{D}}),
$$

where $\mathrm{M}^{*}(\overline{\mathcal{D}})$ is the number of good tilings of $\overline{\mathcal{D}}$. Indeed, the expression on the right of (14) is exactly the number of perfect matchings of the graph obtained from the dual graph of $\overline{\mathcal{D}}$ by removing all its vertical edges corresponding to bad dominoes, i.e. the graph $G^{\prime}$. 
We have a bijection between the set of good tilings of $\overline{\mathcal{D}}$ and the set of $w$-tuples of non-intersecting Schröder paths $\left(\tau_{1}, \tau_{2}, \ldots, \tau_{w}\right)$ compatible with the barriers of $\overline{\mathcal{D}}$, where $\tau_{i}$ starts by the center of the $i$ th vertical step (from bottom) on the southwestern boundary, and ends by the center of the $i$ th vertical step on the southeastern boundary of $\overline{\mathcal{D}}$ (illustrated in Figure 13). In particular the bijection works as in the next paragraph.

It is easy to see that each good tiling of $\overline{\mathcal{D}}$ gives a unique $w$-tuple of non-intersecting paths $\left(\tau_{1}, \tau_{2}, \ldots, \tau_{w}\right)$. On the other hand, given a $w$-tuple of non-intersecting paths $\left(\tau_{1}, \tau_{2}, \ldots, \tau_{w}\right)$, we can recover the corresponding good tiling of the region as follows. The up and down steps in each path $\tau_{i}$ are covered by vertical dominos, and the level steps are covered by horizontal dominos. After covering all steps of all paths $\tau_{i}$ 's, we cover the rest of the region by horizontal dominos.

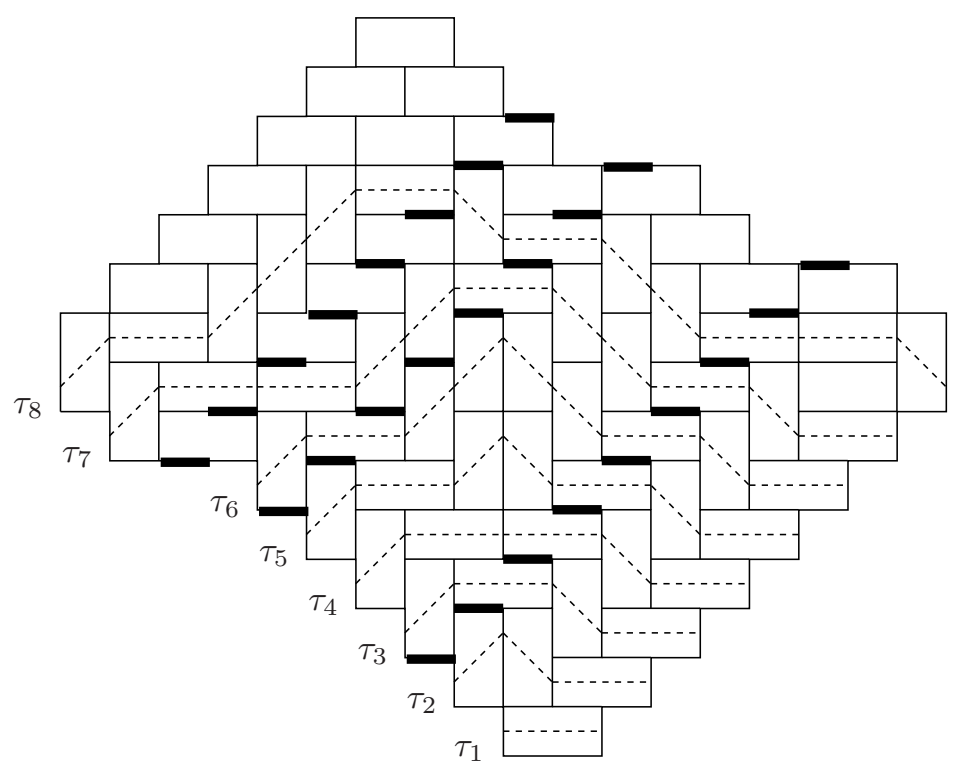

Figure 13: Bijection between domino tilings and non-intersecting paths

Next, we have a bijection between the set of $w$-tuples $\left(\tau_{1}, \tau_{2}, \ldots, \tau_{w}\right)$ above and the set $\Pi_{w}\left(a_{1}, \ldots, a_{k-1}\right)$ of $w$-tuples $\left(\pi_{1}, \pi_{2}, \ldots, \pi_{w}\right)$ (shown in Figure 14). Precisely, the Schröder path $\pi_{i}$ is obtained from $\tau_{i}$ by adding $i-1$ up steps before its starting point, and adding $i-1$ down steps after its ending point, i.e. $\pi_{i}:=\mathrm{U}^{(i-1)} \tau_{i} \mathrm{D}^{(i-1)}$, for $i=1,2, \ldots, w$.

By the two above bijections and (14), we get (13).

We are now ready to prove Theorem 4.

Proof of Theorem 4. We prove (2) by induction on the number of layers $k$ of the region.

For $k=1$, the region $D_{a}\left(d_{1}, \ldots, d_{k}\right)$ is the Aztec diamond of order $a$, so (2) follows from the Aztec diamond theorem 1.

For the induction step, suppose (2) holds for any generalized Douglas regions with strictly less than $k$ layers, for some fixed $k \geqslant 2$. We need to show that (2) holds for any generalized Douglas region $D_{a}\left(d_{1}, \ldots, d_{k}\right)$. 


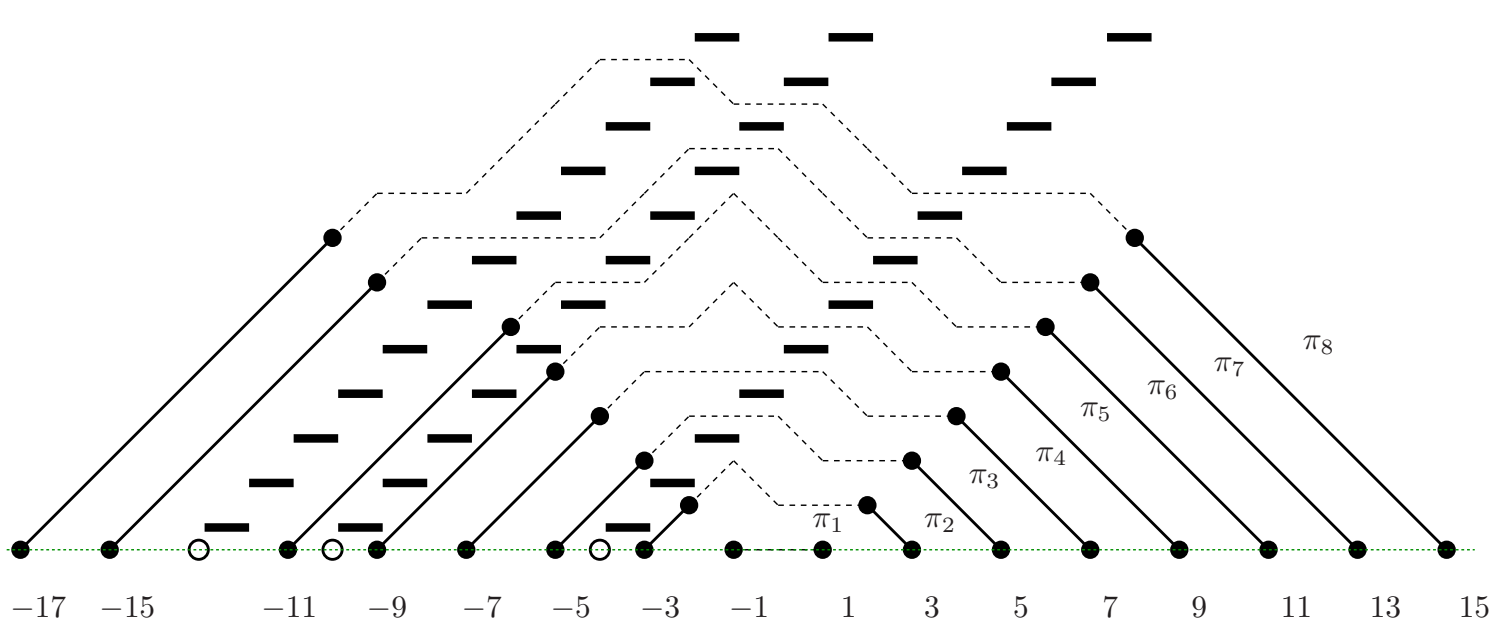

Figure 14: Bijection between $\left(\tau_{1}, \ldots, \tau_{8}\right)$ in Figure 13 and $\left(\pi_{1}, \ldots, \pi_{8}\right)$

Let $a_{i}:=d_{k}+\ldots+d_{k-i+1}+i-1$, for $i=1,2, \ldots, k-1$, as in Proposition 9. Recall that we denote by $p, q, l$ the numbers of lines of black squares, of black up-pointing triangles, and of black down-pointing triangles, respectively.

There are two cases to distinguish, based on the parity of $d_{k}$.

Case I. $d_{k}$ is even.

Assume that $d_{k}=a_{1}=2 x$, for some $x \geqslant 1$. The last layer of the region has $x$ lines of black square, so $p \geqslant x$; and the $(k-1)$ th layer has a line of black up-pointing triangles with bases resting on the last drawn-in diagonal, so $q \geqslant 1$. Thus, $w=p+q \geqslant x+1$ (by $(4))$.

By Proposition 9, we have

$$
\mathrm{M}\left(D_{a}\left(d_{1}, \ldots, d_{k}\right)\right)=\left|\Pi_{w}\left(a_{1}, \ldots, a_{k-1}\right)\right| .
$$

By Propositions 6 and 7, we obtain

$$
\left|\Pi_{w}\left(a_{1}, \ldots, a_{k-1}\right)\right|=\operatorname{det}\left(H_{w}\right)=2^{w} \operatorname{det}\left(G_{w}\right)=2^{w}\left|\Lambda_{w}\left(a_{1}, \ldots, a_{k-1}\right)\right| .
$$

We apply Proposition 8(a) and obtain

$$
\left|\Lambda_{w}\left(a_{1}, \ldots, a_{k-1}\right)\right|=\left|\Pi_{w-1}\left(a_{1}-2, \ldots, a_{k-1}-2\right)\right| .
$$

Two equalities (16) and (17) imply

$$
\left|\Pi_{w}\left(a_{1}, \ldots, a_{k-1}\right)\right|=2^{w}\left|\Pi_{w-1}\left(a_{1}-2, \ldots, a_{k-1}-2\right)\right| .
$$

We apply (18) $x$ times, obtain

$$
\left|\Pi_{w}\left(a_{1}, \ldots, a_{k-1}\right)\right|=2^{\sum_{i=0}^{x-1}(w-i)}\left|\Pi_{w-x}\left(0, a_{2}-a_{1} \ldots, a_{k-1}-a_{1}\right)\right| .
$$

By equality (3), we have $a=p+l \geqslant p \geqslant x$. There are now two subcases to distinguish, depending on the value of $a$. 
Case I.1. $a=x$.

The equality (3) implies $p=x$ and $l=0$. By (7), we have $q=k-1$; and by (6), we obtain

$$
a+w=2 p+n+m=2 x+k-1=\sum_{i=1}^{k} d_{i} .
$$

Since $d_{k}=2 x$, we have $d_{1}=d_{2}=\ldots=d_{k-1}=1$ (see Figure 15 for an example of the generalized Douglas region in this case). Moreover, by (4), we get $w=p+q=x+k-1$. It is easy to see that

$$
\left|\Pi_{w-x}\left(0, a_{2}-a_{1} \ldots, a_{k-1}-a_{1}\right)\right|=\left|\Pi_{k-1}(0,2,4, \ldots, 2(k-1))\right|=1,
$$

SO

$$
\mathrm{M}\left(D_{a}\left(d_{1}, \ldots, d_{k}\right)\right)=2^{\sum_{i=0}^{x-1}(w-i)} .
$$

One can verify that $\mathcal{C}=(w+1) x+\sum_{i=0}^{w-a-1}(w-i)$. Since $a=x$, the equation (2) follows.

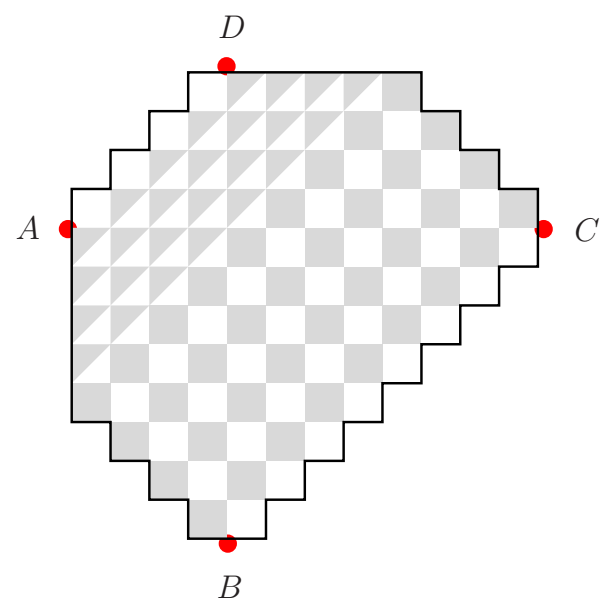

Figure 15: Illustrating the region in Case I.1.

Case I.2. $a>x$.

Then there is some $d_{i}>1$, for some $1 \leqslant i \leqslant k-1$, so the $i$ th layer has at least one line of black squares. Since the last layer still have $x$ lines of black squares, we have $p \geqslant x+1$. Since we already have $q \geqslant 1$ from the argument at the begining of Case I, $w=p+q \geqslant x+2$. By Proposition 8(c), we get

$$
\left|\Pi_{w}\left(a_{1}, \ldots, a_{k-1}\right)\right|=2^{\sum_{i=1}^{x-1}(w-i)}\left|\Pi_{w-x-1}\left(a_{2}-a_{1}-2 \ldots, a_{k-1}-a_{1}-2\right)\right| .
$$

Consider a new generalized Douglas region $\mathcal{D}^{\prime}:=D_{a-x}\left(d_{1}, \ldots, d_{k-1}-1\right)$ having $k-1$ layers. Assume that $\mathcal{C}^{\prime}$ is the number of black regular cells in $\mathcal{D}^{\prime}$, and $w^{\prime}$ is the width of $\mathcal{D}^{\prime}$. Intuitively, $\mathcal{D}^{\prime}$ is obtained from $\mathcal{D}$ by removing its last layer and the line of black up-pointing triangles right above the last layer, and reducing the length of all remaining 
lines of cells by $x$ units (see Figure 16 for an example). Therefore, one can see that $w-w^{\prime}=x+1$, and $\mathcal{C}-\mathcal{C}^{\prime}=(w+1) x+w+x(w-x-1)$. Thus, by induction hypothesis

$$
\mathrm{M}\left(\mathcal{D}^{\prime}\right)=2^{\mathcal{C}^{\prime}-w^{\prime}\left(w^{\prime}+1\right) / 2}=2^{\mathcal{C}-(w+1) x-w-x(w-x-1)-(w-x-1)(w-x) / 2} .
$$

Moreover, by Proposition 9, we get

$$
\mathrm{M}\left(\mathcal{D}^{\prime}\right)=\left|\Pi_{w-x-1}\left(a_{2}-a_{1}-2 \ldots, a_{k-1}-a_{1}-2\right)\right| .
$$

By (15), (20), (21) and (22), we obtain

$$
\begin{aligned}
\mathrm{M}\left(D_{a}\left(d_{1}, \ldots, d_{k}\right)\right) & =2^{\sum_{i=0}^{x-1}(w-i)} \mathrm{M}\left(\mathcal{D}^{\prime}\right) \\
& =2^{\sum_{i=0}^{x-1}(w-i)} 2^{\mathcal{C}-(w+1) x-w-x(w-x-1)-(w-x-1)(w-x) / 2},
\end{aligned}
$$

which implies (2).

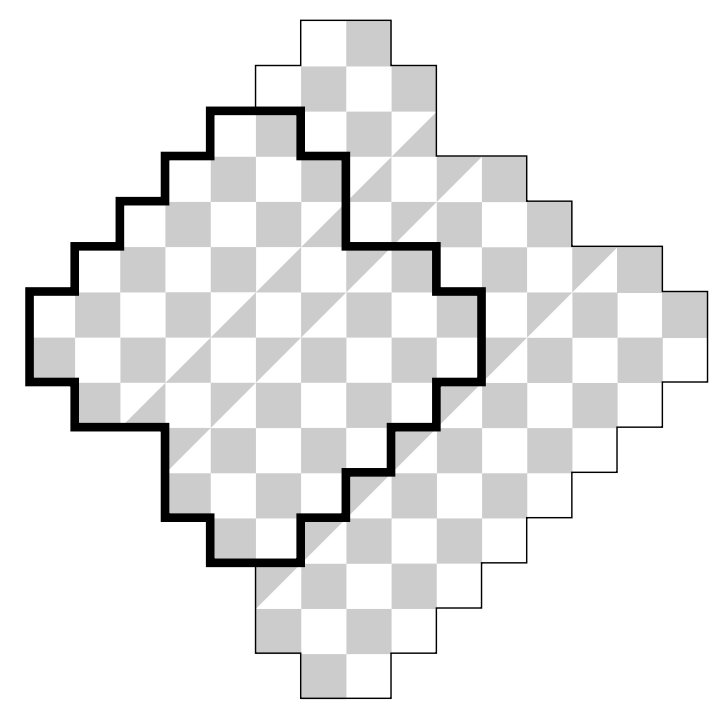

Figure 16: Comparison of two regions $\mathcal{D}=D_{7}(4,2,5,4)$ and $\mathcal{D}^{\prime}=D_{5}(4,2,4)$ (restricted by the bold contour).

Case II. $d_{k}$ is odd.

Assume that $d_{k}=a_{1}=2 x+1$, for some $x \geqslant 0$. By (5b), (6), and (7), we have

$$
2 p+k-1=2 p+m+n=\sum_{i=1}^{k-1} d_{i}+2 x+1 \geqslant 2 x+1+k-1 .
$$

Thus, $p \geqslant x+1$, and by (4), we imply $w=p+q \geqslant x+1$. Note that the last layer has now one line of black down-pointing triangles right below the last drawn-in diagonal. Thus, $l \geqslant 1$, and by (3), $a=p+l \geqslant x+2$. 
We have also the two equalities (15) and (18) as in Case 1. We apply (18) $x$ times, and obtain

$$
\left|\Pi_{w}\left(a_{1}, \ldots, a_{k-1}\right)\right|=2^{\sum_{i=0}^{x-1}(w-i)}\left|\Pi_{w-x}\left(1, a_{2}-a_{1}+1 \ldots, a_{k-1}-a_{1}+1\right)\right| .
$$

By Propositions 6 and 7, we have

$$
\mathrm{M}(\mathcal{D})=2^{\sum_{i=0}^{x-1}(w-i)} 2^{w-x}\left|\Lambda_{w-x}\left(1, a_{2}-a_{1}+1 \ldots, a_{k-1}-a_{1}+1\right)\right| .
$$

There are also two subcases to distinguish, based on the value of $w$.

Case II.1. $w=x+1$.

By (4), we have $q=0$ and $p=x+1$. The equality (24) implies that $\sum_{i=1}^{k-1} d_{i}=k$. Moreover, if $d_{i}=2$ for some $1<i \leqslant k-1$, then the $(i-1)$ th layer has a line of up-pointing triangles with bases resting on the $i$ th drawn-in diagonal, a contradiction to the fact that $q=0$. Therefore, we must have $d_{1}=2$ and $d_{2}=d_{3}=\ldots=d_{k-1}=1$ (see Figure 17 for an example of this case).

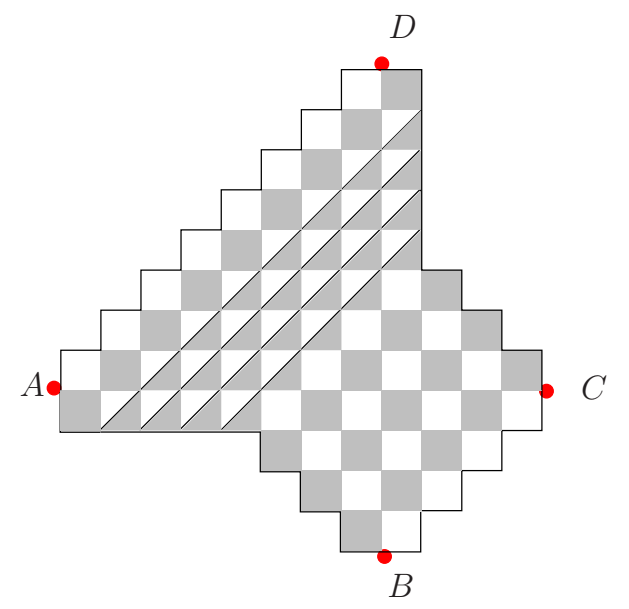

Figure 17: Illustrating the region in Case II.1

We have now

$$
\left|\Lambda_{w-x}\left(1, a_{2}-a_{1}+1 \ldots, a_{k-1}-a_{1}+1\right)\right|=\left|\Lambda_{1}(1,3,5, \ldots, 2 k-3)\right| .
$$

One readily sees that the path in $\Lambda_{1}(1,3,5, \ldots, 2 k-3)$ connecting $(-2 k+1,0)$ to $(1,0)$, so that it never go up once goes down. Thus, we can partition $\Lambda_{1}(1,3,5, \ldots, 2 k-3)=$ $\bigcup_{i=1}^{k} S_{i}$, where $S_{k}$ is the set of paths starting by exactly $i$ up steps followed by down and level steps. It is easy to see that $\left|S_{i}\right|$ is the number of lattice paths using $(1,-1)$ and $(2,0)$ steps from $(-2 k+1+i, i)$ to $(0,1)$ (see Figure 18 for an example with $k=6$; the black dots indicate the points $(-2 k+1+i, i)$ 's). Thus, $\left|S_{i}\right|=\left(\begin{array}{c}k-1 \\ i-1\end{array}\right)$, for $1 \leqslant i \leqslant k$, and $\left|\Lambda_{w-x}\left(1, a_{2}-a_{1}+1 \ldots, a_{k-1}-a_{1}+1\right)\right|=\left|\Lambda_{1}(1,3,5, \ldots, 2 k-3)\right|=\sum_{i=1}^{k}\left(\begin{array}{c}k-1 \\ i-1\end{array}\right)=2^{k-1}$. 


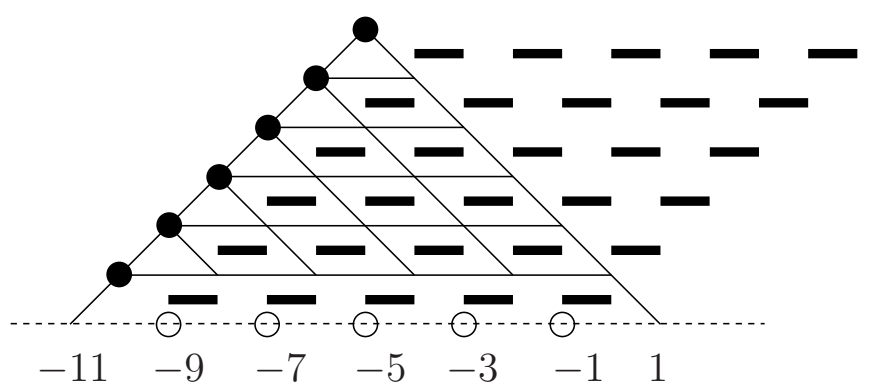

Figure 18: Illustrating all possible steps of the path in $\Lambda_{1}(1,3,5,7,9)$.

By (26), we have $\mathrm{M}(\mathcal{D})=2^{k-1+\sum_{i=0}^{x}(w-i)}$. It is easy to see that $a=w+k-1$ and $\mathcal{C}=(w+1) x+a$, so $(2)$ follows.

Case II. 2. $w>x+1$.

By (26) and Proposition 8(b), we obtain

$$
\begin{aligned}
\mathrm{M}(\mathcal{D}) & =2^{\sum_{i=1}^{x}(w-i)}\left|\Lambda_{w-x}\left(1, a_{2}-a_{1}+1 \ldots, a_{k-1}-a_{1}+1\right)\right| \\
& =2^{\sum_{i=1}^{x}(w-i)}\left|\Pi_{w-x-1}\left(a_{2}-a_{1}-1 \ldots, a_{k-1}-a_{1}-1\right)\right| .
\end{aligned}
$$

Consider the region $\mathcal{D}^{\prime \prime}:=D_{a-x-1}\left(d_{1}, \ldots, d_{k-1}\right)$ (note that we already showed that $a \geqslant$ $x+2$ at the begining of Case II). Assume that $\mathcal{C}^{\prime \prime}$ is the number of regular black cells in $\mathcal{D}^{\prime \prime}$, and $w^{\prime \prime}$ is the width of $\mathcal{D}^{\prime \prime}$. The region $\mathcal{D}^{\prime \prime}$ is obtained from $\mathcal{D}$ by removing its last layer, reducing the length of all remaining lines of cells by $x+1$ units (see the region restricted by the bold contour in Figure 19(a)), and replacing the bottom line of white triangles in the resulting region by a line of white squares (see Figure 19(b)). Therefore, $w-w^{\prime \prime}=x$ and $\mathcal{C}-\mathcal{C}^{\prime \prime}=(w+1) x+(x+1)(w-x)$. By induction hypothesis, we have

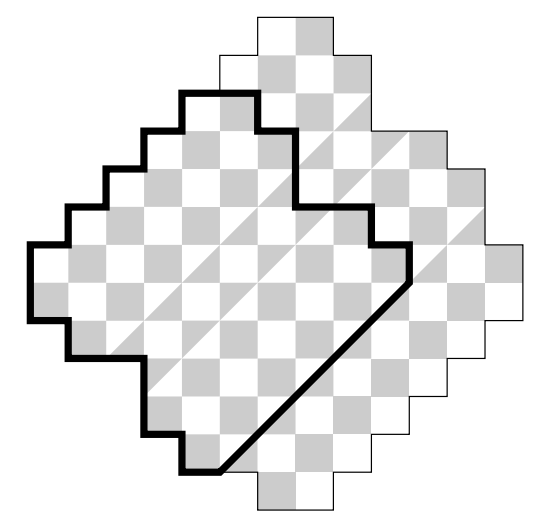

(a)

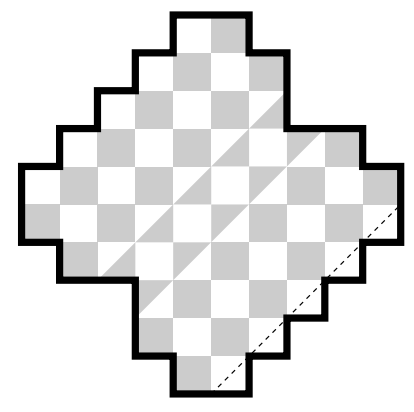

(b)

Figure 19: Comparison of two regions $\mathcal{D}$ and $\mathcal{D}^{\prime \prime}$ (right).

$$
\mathrm{M}\left(\mathcal{D}^{\prime \prime}\right)=2^{\mathcal{C}^{\prime \prime}-w^{\prime \prime}\left(w^{\prime \prime}+1\right) / 2}=2^{\mathcal{C}-(w+1) x-(x+1)(w-x)-(w-x)(w-x+1) / 2} .
$$


Moreover, by Proposition 9, we get

$$
\mathrm{M}\left(\mathcal{D}^{\prime \prime}\right)=\left|\Pi_{w-x-1}\left(a_{2}-a_{1}-1 \ldots, a_{k-1}-a_{1}-1\right)\right| .
$$

Finally, by (27), (28) and (29), we obtain

$$
\begin{aligned}
\mathrm{M}\left(D_{a}\left(d_{1}, \ldots, d_{k}\right)\right) & =2^{\sum_{i=0}^{x}(w-i)} \mathrm{M}\left(\mathcal{D}^{\prime \prime}\right) \\
& =2^{\sum_{i=0}^{x}(w-i)} 2^{\mathcal{C}-(w+1) x-(x+1)(w-x)-(w-x)(w-x+1) / 2},
\end{aligned}
$$

which implies (2).

\section{Concluding remarks}

We have two other proofs of the main theorem, Theorem 4, using Kuo's graphical condensation [10] and a certain reduction theorem due to Propp [12], respectively. We are planning to present them in a subsequent paper.

\section{References}

[1] M. Aigner. A course in Enumeration. Springer Press, 2007.

[2] F. Bosio and M. A. A. van Leeuwen. A bijection proving the Aztec diamond theorem by combing lattice paths Elec. J. Combin., 20(4): \#P24, 2013.

[3] R. Brualdi and S. Kirkland. Aztec diamonds and digraphs, and Hankel determinants of Schrder numbers. J. Combin. Theory Ser. B, Vol. 94, Issue 2: 334-351, 2005

[4] C. Douglas. An illustrative study of the enumeration of tilings: Conjecture discovery and proof techniques, 1996. Available at: http://citeseerx.ist.psu.edu/viewdoc/summary?doi=10.1.1.44.8060

[5] N. Elkies, G. Kuperberg, M. Larsen, and J. Propp. Alternating-sign matrices and domino tilings. J. Algebraic Combin., 1: 111-132, 219-234, 1992.

[6] S.-P. Eu and T.-S. Fu. A simple proof of the Aztec diamond theorem. Elec. J. Combin., 12: \#R18, (2005).

[7] I. M. Gessel and X. Viennot. Binomial determinants, paths, and hook length formulae. Adv. in Math., 58: 300-321, 1985.

[8] I. M. Gessel. Schröder numbers, large and small. A talk at CanaDAM 2009. Slide is available at: http://people.brandeis.edu/ gessel/homepage/slides

[9] S. Kamioka Laurent biorthogonal polynomials, $q$-Naryana polynomials and domino tilings of the Aztec diamonds, J. Combin. Theory Ser. A 123, 2004, 14-29.

[10] E. H. Kuo. Applications of graphical condensation for enumerating matchings and tilings. Theoretical Computer Science, 319: 29-57, 2004. 
[11] B. Lindström. On the vector representations of induced matroids. Bull. London Math. Soc., 5: 85-90, 1973.

[12] J. Propp. Generalized domino-shuffling, Theoretical Computer Science, 303: 267-301, 2003.

[13] J. R. Stembridge. Nonintersecting paths, Pfaffians and plane partitions. Adv. in Math., 83: 96-131, 1990. 